\title{
¿Quién soy? Reconsiderando nuestro papel en la comunidad. Co-creando el sentimiento de identidad ${ }^{1}$
}

\author{
Silvia Jiménez Torres ${ }^{2}$ \\ $I P R, I A R P P, I P S A$-Levante
}

En este trabajo se presentan tres casos clínicos dónde se muestra cómo los valores, creencias, visión del mundo, costumbres, roles de género, etc. transmitidos inter-generacionalmente en la comunidad de la que somos parte, influyen en la formación del sentimiento de identidad. Ante esto, "...el terapeuta no ha de permanecer en silencio, sino que es nuestra responsabilidad comprometernos en la acción transformadora, revisando para ello, nuestra posición como analistas-sujetos sociales que vivimos en una época, sociedad y contexto concreto" (Ávila Espada, 2016).Se consideran tres aspectos para la compresión de estos tres casos clínicos: el desfondamiento, la teoría del apego y la cultura.

Palabras clave: Comunidad, sentimiento de identidad, desfondamiento, teoría del apego, cultura.

In this paper, three clinical cases are presented, showing how values, beliefs, world views, customs, gender roles, etc. transmitted intergenerationally in the community of which we are part, influence the formation of the feeling of identity. Given this, "... the therapist should not remain silent, but it is our responsibility to commit ourselves to the transformative action, reviewing for this our position as analysts-social subjects that live in a specific time, society and context" (Ávila Espada, 2016). Three aspects are considered for the understanding of these three clinical cases: breaking up, the theory of attachment and culture.

Key Words: Community, feeling of identity, breaking up, theory of attachment, culture.

English Title: Who I am? Reconsidering our role in the community. Co-creating the feeling of identity

\section{Cita bibliográfica / Reference citation:}

Jiménez Torres, S. (2018). ¿Quién soy? Reconsiderando nuestro papel en la comunidad. Cocreando el sentimiento de identidad. Clínica e Investigación Relacional, 12 (1): 88-96. [ISSN 1988-2939]

[Recuperado de www.ceir.info ] DOI: 10.21110/19882939.2018.120107

\footnotetext{
${ }^{1}$ Este trabajo ha sido leído como comunicación en las V Jornadas de Psicoanálisis Relacional, organizadas por el Instituto de Psicoterapia Relacional en el Real Sitio de La Granja de San Ildefonso (Segovia), los días 23 y 24 de febrero de 2018-

2 Doctora en Psicología. Licenciada en Filosofía y Ciencias de la educación -sección psicología- (Universidad de Salamanca). D.E.A en Neuropsicología clínica (Universidad de Salamanca), Diplomada en Magisterio (Universidad de Zaragoza). Máster en integración de personas con discapacidad (Universidad de Salamanca). Psicoterapeuta de orientación psicoanalítica. Miembro de IARPP-España y de IARPP-Internacional. Miembro de IPR. Directora de formación de IPSA-Levante. Dirección profesional: C/ Orihuela, nº 36, pta. 3, 46009, Valencia E-mail: ipsaformacion@gmail.com
} 
Antes de comenzar con la exposición de los tres casos clínicos me gustaría leer una cita de Joan Coderch y Alejandra Plaza (2016, p.125-126) que considero nos prepara para el análisis posterior de los casos: "...desde el mismo momento del nacimiento, el bebé y quienes le rodean, forma un sistema intersubjetivo, dinámico, abierto y no lineal que constituye la matriz relacional y socio/cultural [...] hasta que muy lentamente [...] los seres humanos salimos materialmente de la matriz relacional y socio/cultural que nos ha acogido, pero ella no sale de nuestro interior".

Se consideran tres aspectos para la compresión de estos tres casos clínicos: el desfondamiento, la teoría del apego y la cultura, a sabiendas de que hay muchos otros factores intervinientes, pero que por razones de tiempo no es posible desarrollar en este momento.

La primera viñeta clínica que presentaré es la de Raquel. Es una mujer de 35 años. Comenzó psicoterapia conmigo en octubre de 2015 aquejada de "depresión". En ese momento me comunicó que "hace 304 años caí en la depresión".

Raquel es enfermera, vive en un pueblo de fuera de la Comunidad Valenciana y trabaja con personas mayores en un hospital también fuera de Valencia. Vive en el mismo pueblo que sus padres y dónde pasó toda su infancia. Tiene un hermano mayor, casado y con 2 hijos que viven en un lugar diferente al pueblo de Raquel. En el momento en que comenzó terapia tenía una pareja, sin ser pareja (en sus propias palabras). Él no quería una relación seria, de pareja. Él vive en una ciudad diferente, y, en palabras de Raquel, cuando se veían allí, sí parecían una pareja. Sin embargo, en el pueblo, era diferente, como un amigo más, siempre y cuando, claro está, estuvieran con gente. Cuando quería encuentros sexuales, se volvía a comportar como pareja. Ella, al principio, asumió esto, aunque con el tiempo esa ambivalencia le producía profundos sentimientos de pena y tristeza.

En las primeras sesiones cuenta que estuvo siete años viviendo en Valencia, mientras estudiaba, pero cuando regresó "mi madre ya estaba depresiva" y en ese momento "dejé de hacer lo que yo quería". Además, la madre tiene una enfermedad que la ha ido incapacitando poco a poco, aunque en el momento de comenzar terapia, no era muy acusado. Sin embargo, los padres fueron a vivir a casa de Raquel.

Como la madre estaba depresiva, ella pensaba que su madre la necesitaba y antepuso todas sus necesidades a las suyas propias. Estuviera haciendo, lo que estuviera haciendo, Raquel llamaba a su madre cada dos horas. Si no lo hacía era la madre la que la llamaba, muy enfadada, y si no le cogía el teléfono llamaba insistentemente una y otra vez, hasta poder encontrarse en una hora 9 llamadas perdidas. 
Refiere en las primeras entrevistas que cree que ya estaba anulada anteriormente, aunque en ese momento no se daba cuenta. Siempre ha tenido claro que su responsabilidad era hacerse cargo de sus padres, aunque fuera a costa suya, ya que es lo que le toca. Desde niña ha cumplido con todo, con los estudios, con el trabajo, con los padres, con el hermano, con los amigos..., porque es lo que se espera de ella.

Cuenta que ya con 10012 años, se tenía que encargar de sus abuelos. Si había una boda, una comunión, etc. ella no podía asistir, ya que tenía que cuidar de los abuelos, mientras sus padres y su hermano sí iban a la celebración. Con 7 u 8 años ella les pasaba la comida a los abuelos desde su casa a la de ellos. Recuerda una vez en que les pasó la comida y la abuela la probó. Entonces, el abuelo le pegó un bofetón delante de Raquel. La abuela dejó de comery ella regresó a su casa todo el camino llorando. Sus padres no hicieron nada y comenta "A mi abuelo nadie le ha plantado cara". En esos momentos "aprendí que por el hombre hay que aguantar todo". Sin embargo, en su casa, el padre es el más empático y con el que tiene mejor feeling, porque dice "nunca se mete en la vida de los demás y no impone su criterio". Su padre siempre es el que ha hecho todo en casa y contaba cómo agasajaba a su madre. Sin embargo, la madre es posesiva, autoritaria y manipuladora. Cuenta cómo una vez, el hermano se iba a ir de viaje a Roma a ver al Papa con unos amigos y haciéndole chantaje emocional y "poniéndole la cabeza como un bombo", consiguió que el hermano anulara el viaje.

Como decía anteriormente, el hermano y su familia viven en otra ciudad. Sin embargo, tienen una casa encima de la de Raquel para cuando van al pueblo. Contaba, de manera muy emotiva y sentida, cómo durante muchos años, en las fiestas navideñas, mientras toda la familia estaba tomando un aperitivo, ella tenía que encargarse de la comida, la cena, las compras, etc. Además, ella también es la responsable del coste económico. Lo mismo ocurría cuando eran las fiestas del pueblo. Del mismo modo, cuando llegan las vacaciones de verano, el hermano y su familia van al pueblo. Pero en su casa no tenían lavadora ni cocina acondicionada, así que era Raquel, la que les lavaba la ropa, y preparaba la comida para todos. Durante años estuvo sin poder disfrutar de las vacaciones porque tenía que hacerse cargo de sus sobrinos, incluso rechazó un viaje a México porque tenía que quedarse con ellos.

Aunque ella sentía que había algo que no estaba bien, que sentía que se estaban aprovechando de ella, lo que le llevó a una profunda tristeza, a un sentimiento de abatimiento difícil de soportar y a no poder disfrutar de nada, lo asumía como parte de sus funciones, porque si no lo hacía se sentía culpable por no "ayudar" a su familia. Como afirman Coderch y Plaza (2016, p. 256), no podemos olvidar que "[...] los valores por los que se ha regido la familia han sido un espejo de la sociedad y la cultura de la que forma parte". 
A raíz del proceso de psicoterapia, Raquel empezó a modificar sus creencias en cuanto a su papel en la comunidad y en la familia. Pero esto, al principio, no fue bien recibido hasta el punto de que un día los sobrinos le dijeron que sus padres pensaban que estaba descuidando su papel como tía. Su respuesta fue: "Decirles que ya se pueden ir acostumbrando".

Juntas, a través de la relación, estamos reconsiderando su papel en la comunidad y estamos en el camino de co-crear su sentido de identidad.

La segunda viñeta clínica es la de Alba. Cuando comenzó la terapia Alba tenía 28 años. También es enfermera y tiene un hermano mayor, casado y con una hija. Acude a terapia aquejada de un problema de inseguridad que le hace tener ansiedad. Refiere que en situaciones que no controla le entra ansiedad y pánico y dice que reconoce que son reacciones desmesuradas en cuanto a la causa. Sin embargo, en su trabajo, que requiere de respuestas rápidas, certeras y con riesgo de vida o muerte, es muy segura, se siente capacitada y no duda, porque dice ella "es un ámbito que controlo". "Si mis padres me vieran en el trabajo no se lo creerían. Pensarían que no soy yo". También dice que es un poco hipocondríaca.

Cuenta que los padres son muy sobreprotectores y conservadores en el sentido "más vale malo conocido que bueno por conocer". Desde niña recuerda que ella no requería atención, porque era muy buena, siempre se portaba bien porque "yo no podía permitir portarme mal. Yo tenía que sacar buenas notas. Era tan grande la responsabilidad en bachillerato que lo pasé fatal". Fue al psiquiatra y le dijo que tenía ansiedad patológica. Cuenta que su hermano es un caprichoso y egoísta y recuerda que ha estado siempre muy mimado por sus padres y la familia del padre. Además, dice que como el hermano fue el primer hijo y de niño estaba enfermo, ella tuvo que aprender a no molestar. Ha hecho siempre lo que le han dicho los padres, ha cumplido con su obligación, ha obedecido y ha tomado por suyas las decisiones que los padres tomaban por ella, como por ejemplo comprarse un piso. Dice que lo compró porque los padres le dijeron que era un buen momento y que tenía que "meterse" en un piso. Ella está contenta por tener piso, pero refiere que piensa que no ha sido decisión suya, sino de sus padres. Ya de adulta, tenía dificultades para elegir entre comprarse un sueter rojo o verde (por ejemplo) y se siente mejor cuando alguien le dice cual debe comprarse.

Ella ha mediado desde niña en las disputas entre los padres, sobre todo era la madre la que le contaba las discusiones con el padre. Del mismo modo, ha sido también el contenedor donde su madre se desahoga con respecto al hermano.

Algo repetido a menudo al comienzo de la terapia era su temor a ir a casa de los padres o a que ellos fueran a su casa. Siempre que ocurría tenía dolor de cabeza. Refería que eso sucedía porque cuando los padres van a su casa nunca están a su gusto: "o no está lo suficientemente 
recogida, o me indican que tengo que fregar la vajilla, o las plantas no están como deberían..." y si es ella la que va, siempre hay algo que reprocharle, o no va las suficientes veces, o no está el tiempo suficiente, o no opina lo mismo que ellos... Ella dice: "Siempre se están metiendo conmigo. Muchas veces siento que se divierten ridiculizándome. A menudo me repiten que soy torpe". Sus opiniones son cuestionadas, siempre y cuando no coincidan con las de sus padres. Siente "que haga lo que haga nunca está bien ni es suficiente". Agravado porque "con mi padre nunca sabes por dónde te va a salir". Como ejemplo, cuenta que unas Navidades, ella trabajaba la Noche Buena por la noche. Al día siguiente comían todos en casa de sus padres y Alba llegó un poco justa de tiempo, ya que cuando llegó de trabajar tuvo que acostarse a dormir un poco. El padre la trató con desprecio, no le hablaba, la miraba mal, pero sin decir que le pasaba algo con ella y por qué estaba enfadado. Alba dice: "Llegué justa porque había estado toda la noche trabajando, no porque estuviera de fiesta. Una prima mía llegó más tarde que yo y no pasó nada. Pero conmigo sí".

Dice que siempre ha habido diferencias entre el hermano y ella. Por ejemplo, recuerda que cuando la abuela materna les hacía algún regalo, al hermano le llevaba, por ejemplo, unas zapatillas de 20.000 pts y a ella un paquete de galletas. Siente que el hermano ha sido el ojo derecho de la abuela paterna y de la materna y también de los padres y que ella solo ha sido especial para su abuelo materno.

Recuerda que en casa de sus padres "siempre ha habido abuelos", la madre se ha encargado tanto de los suegros como de los padres. Ella también afirmaba que cuando sus padres sean mayores tendrá que ocuparse de ellos porque es lo que le toca.

Todo esto, entre otras cosas, ha llevado a Alba a tener miedo al abandono, a que no la tengan en cuenta, a que la dejen de lado, y hace lo que sea necesario para que esto no ocurra. Por ejemplo, Alba es muy activa socialmente y tiene diferentes grupos de amigos. En cualquier reunión social Alba procura que todas las personas se sientan a gusto, aunque para ello ella no pueda disfrutar. Así se siente dependiente de las personas cercanas y junto con la inestabilidad en su autoimagen y el temor al abandono, su capacidad de decisión se ve disminuida (si no actúa, piensa y decide según lo que se espera de ella, pueden abandonarla).

Alba convive con su pareja, David, y es con él con quien manifestaba de manera más acusada su inseguridad y su temor al abandono. Sin embargo, él es la persona que más apoya a Alba en sus decisiones, sin cuestionarlas. En palabras de Alba : "David me hace la vida fácil". Pero David, es algo más joven que ella y también más inmaduro, lo que fomentaba que Alba se pusiera nerviosa si salía y no le avisaba de dónde estaba. También era Alba la que le daba las consignas y le recordaba si tenía que apagar la luz, si tenía que cerrar las puertas... o cómo debía comportarse en según qué situaciones. 
Durante un tiempo de la terapia, Alba manifestaba no estar del todo a gusto con su trabajo en un hospital privado y su deseo de intentar cambiar a la sanidad pública, ya que esto le suponía una mejora tanto económica como de posibilidades futuras, pero no se atrevía. A raíz del proceso de la psicoterapia, Alba cambió hace unos meses de trabajo, con unos resultados muy positivos. Ha cambiado su percepción de sí misma, ha flexibilizado el control de su pareja, está más segura de las decisiones que toma y es capaz, con su familia de defender sus opiniones con asertividad. Sin embargo, este hecho hace que los padres le digan que desde hace unos meses está un poco contestona, lo que, en un principio, le hacía dudar de sí misma. Sin embargo, esto ha cambiado, y en estos momentos ha aparecido una nueva visión del mundo para ella, dándole la posibilidad de actuar y resolver problemas de formas que antes no se habían ejercitado y tiene una mayor comprensión de sí misma, fortaleciendo así su capacidad de agencia.

La tercera viñeta clínica es la de Lucía. Lucía tenía 29 años cuando comienza terapia. Es enfermera y trabaja en un hospital. Viene aquejada de ansiedad y problemas en el sueño y unos altos niveles de tristeza y desmotivación. También presenta trastornos de la alimentación. Vive independizada de su familia desde que se casó hace 8 años, aunque hace 2 que se divorció, porque su marido y la familia de él le estafaron y perdió todos sus ahorros. La familia de su exmarido la trató muy mal porque no se dejó estafar más todavía, aunque en ese momento ella hacía cualquier cosa por él.

Sus padres viven cerca de ella, y el padre tiene cáncer desde hace varios años que le ha dejado bastante incapacitado. Es Lucía, junto con la madre, la que se encarga básicamente de su cuidado (aunque no viva con ellos). Tiene un hermano menor que ella, casado y padre de una niña de 18 meses que vive en otra ciudad y que no se preocupa de la enfermedad del padre "es como si no existiera la enfermedad". De hecho, cuando necesitan ayuda en el cuidado de la niña, llaman a los padres de Lucía y estos se trasladan a la ciudad del hermano para cuidar de la niña. Evidentemente, la que se encarga de la nieta es la madre, ya que el padre está muy enfermo, pero aún así, se trasladan los dos a casa del hermano. Sin embargo, Lucía justifica al hermano porque, dice, le corresponde a ella cuidar de sus padres ya que es mujer, es la hermana mayor y es enfermera. Además, sus padres han reforzado esta idea desde siempre y hasta el día de hoy.

De niña, los padres nunca necesitaron prestarle mucha atención, ya que era una niña muy buena, obediente, estudiosa, responsable... y no así el hermano que, según ella, "era un bicho".

En la tercera sesión conmigo, se sorprende muchísimo y explica que es porque se ha acordado de repente que en la preadolescencia estuvieron a punto de violarla dos veces, con 
una diferencia de días, por la misma persona. Fue en el portal de la finca donde vivía, y las dos veces consiguió que el asaltante huyera al ponerse a gritar. Ya había logrado el violador hacerlo con otras personas de las fincas cercanas. Lucía dice que lo peor de todo es que la primera vez los padres no le dieron importancia y no hicieron nada. La segunda vez, alertados por los vecinos, el padre salió a la calle con una escopeta para matar al violador. Lucía decía que ninguna de las dos cosas le había resultado de ayuda.

Desafortunadamente, Lucía ha tenido que interrumpir el tratamiento por circunstancias personales.

El ser humano nace desfondado porque la naturaleza no le ha dotado de un repertorio de instintos y reglas biológicas para organizar su vida, su sociedad y saber cómo actuar; para fondarse necesita ser-estar-en el mundo. Nacemos con un enorme potencial para el establecimiento de relaciones, pero no estamos programados para tratar eficazmente nuestro mundo emocional interno ni el relacional con nuestros congéneres. Buscamos "fondarnos" en los demás y también a través de la cultura, sus funciones y sus símbolos (Luís Cencillo, 1978).

Siguiendo a Luís Cencillo (1978, p. 604), desde la ontología, podemos decir que el hombre tiene que hacerse a través de su historia, en su proceso histórico individual, ya que no le viene dada su condición como a una cosa. No existe un fondo de ideas y de tablas de valores, dado automáticamente, para sostener nuestras opciones, pero sí existe la praxis y nuestra vida concreta desde la cual conocemos, pensamos, sentimos y valoramos. No hay nada en el hombre, ni cuando nos referimos a él como individuo ni como grupo, que no haya sido aprendido o transmitido tradicionalmente por una sociedad, salvo su forma original de combinar y vivir estas tradiciones, lo que no le determina hacia un tipo de comportamiento único. En este sentido, Coderch (2014) también expone que el ser humano es una expresión de una cultura o una mezcla de ellas, es decir, no existen los seres humanos aculturales, provistos únicamente de equipo genético. Sino que,

"[...] después de su salida de la matriz biológica la vida del bebé transcurre en otra matriz, la matriz relacional y socio/cultural constituida por la madre en primer lugar, el padre, los hermanos $y$, posteriormente, un pequeño grupo de otros familiares y cuidadores. Unos y otros pertenecen al mismo grupo cultural y comparten la misma lengua y las mismas costumbres y hábitos, todo lo cual comporta unas maneras similares de tratar a los bebés, las cuales influirán profundamente en la personalidad y curso de la vida del recién nacido." (Coderch y Plaza, 2016, p. 125).

El bebé busca fondarse en la relación con sus principales figuras de apego. Como sabemos, la teoría del apego (Bowlby, 1969) defiende que, desde el nacimiento, el niño es un activo 
buscador de figuras de apego, lo que responde a una necesidad básica original, que favorece la supervivencia de la especie. Las necesidades básicas del bebé están preprogramas para ser satisfechas en sociedad, mientras que el grupo social en el que el niño nace le transmite la cultura de su especie, y también le necesita para perpetuarse, desarrollarse y sobrevivir (Jiménez, 2011). Es en el contexto de las relaciones de apego donde se desarrolla la mentalización, que es crucial para la autorregulación y para las relaciones íntimas constructivas.

Y, en general, las principales relaciones de apego se dan en la familia. Podemos decir, que la familia representa a la sociedad en la que convive, o lo que es lo mismo, la familia es un espejo de la sociedad y la cultura de la que forma parte. Adam Kuper (2001) expone que es participando en la identidad de una colectividad (por ejemplo, una nación, una minoría étnica, una clase social, o un movimiento religioso) donde el yo interior encuentra su hogar en el mundo. Por su parte, Zygmunt Bauman (2017) señala que los conceptos de construcción identitaria y cultura nacieron juntos. Por lo que, una vez se ha establecido una identidad cultural, se produce una presión que empuja a vivirla, incluso aunque eso signifique sacrificar la propia individualidad (Kuper, 2001, p, 272). Y, como señala Guerra "...parte del problema reside en la exigencia de nuestra cultura" (2013, p. 72).

Una de las cosas que podemos observar es que los miembros de la familia tienden a adoptar un estado atractor, es decir, a organizarse de manera que cada miembro de la familia tenga asignado un papel característico incuestionable que, a menudo, pesa sobre ellos como una insoportable carga (Coderch y Plaza, 2016). Raquel, Alba y Lucía, son tres mujeres que forman parte de la misma generación y, podemos observar, con una estructura familiar bastante similar. Las figuras de apego de las tres, han sido las principales, pero no las únicas, transmisoras de los valores, creencias, visión del mundo, costumbres, roles de género, etc. que existen en la comunidad de la que son parte, y les han asignado un papel determinado y similar a cada una de ellas. Desde que recuerdan, las tres, de un modo u otro, han sabido que lo que se espera de ellas es ser buenas, obedientes, responsables, trabajadoras y, sobre todo, ceder abnegadamente en beneficio de los hermanos. $Y$, en las tres nos encontramos lo que se denomina "role reversal", es decir, un intercambio de roles con sus figuras de apego. Algo que se puede entender como un trauma del desarrollo, un trauma relacional que desconfigura el desarrollo natural del self (Guerra, 2018). Han interiorizado su papel de cuidadoras, lo que Gilligan (2013) denomina la ética del cuidado, hasta el punto de llevar a preguntarnos si ha podido influir en su elección profesional.

Las tres han sido víctimas de manipulaciones y chantajes emocionales para mantener ese estado atractor $y$, si en algún momento, alguna de ellas ha intentado rebelarse y hacerse oir, 
se han encontrado con que se las califica de egoístas e insensibles, lo que les hace sentirse culpables y avergonzadas. A lo largo del tiempo, les han hecho sentir que sus opiniones, sus sentimientos y sus deseos no tienen valor, a no ser que coincidan con las de sus figuras de referencia, porque no es lo que se espera de ellas. Y si no tienen valor, no son.

Estos factores han atenuado la voluntad de las tres y la psicoterapia relacional, que privilegia la relación interpersonal, fortalece un sentimiento de self más integrado y auténtico.

\section{REFERENCIAS}

Ávila Espada, A. (2016). Perspectivas relacionales frente al conflicto social: el compromiso del clínico relacional con la acción transformadora. Clínica e Investigación relacional, 10(3):723-727. [ISSN 1988-2939] [Recuperado de www.ceir.info] DOI: $10.21110 / 19882939.2016 .100307$

Bauman, Z. (2017). Modernidad líquida. Madrid, España: Fondo de cultura económica.

Bowlby, J. (1969). Attachment and loss: Vol. 1. Attachment. New York: Basic Books.

Cencillo, L. (1978). El hombre noción científica. Madrid: Pirámide.

Coderch, J. (2014). Avances en Psicoanálisis Relacional. Nuevos campos de exploración para el psicoanálisis. Madrid, España: Ágora Relacional, S.L.

Coderch, J. \& Plaza, A. (2016). Emoción y Relaciones Humanas. El Psicoanálisis Relacional como Terapéutica Social. Madrid, España: Ágora Relacional, S.L.

Gilligan, C. (2013). La ética del cuidado. Cuadernos de la Fundació Víctor Grífols i Lucas, n 30. Barcelona.

Guerra, L.R. (2013). El clavo ardiendo. Claves de las adicciones amorosas y los conflictos en las relaciones de pareja sanas y patológicas. Barcelona, España: Octaedro.

Guerra, L.R. (2018). Palos en las ruedas. Una perspectiva relacional y social sobre por qué el trauma nos impide avanzar. Barcelona, España: Octaedro.

Jiménez, S. (2011). Transferencia y género. En Guerra, L. R. \& Jiménez, S. (Coords), Psicoterapia Psicoanalítica: nuevos caminos para el avance de un método. (193-218). Salamanca, España: Amarú Ediciones.

Kuper, A. (2001). Cultura. La versión de los antropólogos. Barcelona, España: Paidós.

Original recibido con fecha: 17/1/2018 Revisado: 25/1/2018 Aceptado: 28/02/2018 